\title{
INDICACIONES RECURRENTES EN LAS NORMATIVAS PARA EL USO PERIODÍSTICO DE LAS REDES SOCIALES
}

\section{Susana Herrera-Damas}

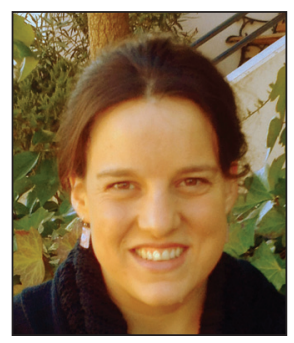

Susana Herrera-Damas es licenciada en comunicación audiovisual (Universidad de Navarra, 1998) y en sociología (UNED, 2004) y doctora en comunicación audiovisual (Universidad de Navarra, 2002). Visiting Scholar en las universidades de Ottawa (Canadá) y Texas (Estados Unidos). Es profesora titular de periodismo en la Universidad Carlos III de Madrid, donde investiga de qué maneras los medios tradicionales pueden acoger mejor los medios sociales para incrementar la calidad del periodismo.

http://orcid.org/0000-0002-1755-1621

Universidad Carlos III de Madrid Calle Madrid, 133 28903 Getafe, Madrid, España dherrera@hum.uc3m.es

\section{Resumen}

En los últimos años, medios de comunicación de todo el mundo están regulando el comportamiento de sus periodistas en las redes sociales, ante el temor de que estas nuevas formas de comunicación comprometan la reputación de sus instituciones. Quienes no lo han hecho contemplan este desafío imparable con cierta preocupación y bosquejan ya las directrices de las que serían sus social media policies. Con el propósito de inspirar el diseño de estas nuevas normativas, se analizan las indicaciones recurrentes que ya han redactado medios de prestigio como AP, American Society of News Editors (ASNE), BBC, Bloomberg, Charlotte observer, Freedom communication, Los Angeles times, NPR, Orlando sentinel, Politico, Reuters, Rockford register star, Saint Louis post-dispatch, Source media group, The Denver post, The guardian, The journal gazette, The Manhattan Mercury, The New York times, The Roanoke times, The Wall Street journal y The Washington post. Para detectar esos temas se ha realizado un análisis cualitativo de contenido de 22 de estas normativas.

\section{Palabras clave}

Redes sociales, Medios, Web 2.0, Normas, Pautas, Guías, Normativas, Periodismo, Periodistas, Ética, Regulación, Autoregulación, Prensa, Radio, Televisión.

\section{Title: Recurrent topics in guidelines for the use of social networks by journalists}

\begin{abstract}
Mainstream media all over the world have been regulating the behavior of their journalists in social media, fearing these new forms of communications might damage the credibility of their institutions. Those who have not yet done so face with concern this unstoppable challenge and are now outlining their own guidelines for social media policies. In an effort to inspire the design of these new policies, this paper aims to approach the recurring topics in the policies already written by leading media such as AP, American Society of News Editors (ASNE), BBC, Bloomberg, Charlotte observer, Freedom communication, Los Angeles times, NPR, Orlando sentinel, Politico, Reuters, Rockford register star, Saint Louis post-dispatch, Source media group, The Denver post, The guardian, The journal gazette, The Manhattan Mercury, The New York times, The Roanoke times, The Wall Street journal y The Washington post. A qualitative content analysis of 22 of these policies has been conducted to identify these topics.
\end{abstract}

\section{Keywords}

Social media policies, Social networks, Web 2.0, Guides, Guidelines, Journalism, Journalists, Ethics, Regulation, Self-regulation, Press, Media, Mass media, Radio, Television.

Herrera-Damas, Susana (2013). “Indicaciones recurrentes en las normativas para el uso periodístico de las redes sociales". El profesional de la información, julio-agosto, v. 22, n. 1, pp. 46-53.

http://dx.doi.org/10.3145/epi.2013.ene.06 


\section{Qué son y para qué sirven las normativas ${ }^{1}$ de actuación en redes sociales}

Las social media policies son documentos que incluyen directrices con las que una empresa espera regular ${ }^{2}$ el comportamiento de sus empleados en las redes sociales ${ }^{3}$. Desde 2009 estas normativas internas son cada vez más frecuentes en los medios de referencia, tanto por el rápido avance de las redes sociales en los últimos años, como por su creciente importancia para obtener información, difundirla y crear comunidad con sus seguidores. Al margen de que no nacieran con esa vocación, las redes sociales contienen un innegable valor periodístico que debería ser estimado ${ }^{4}$, aunque sólo fuera por el hecho de que un número cada vez mayor de políticos, personajes públicos, empresas, instituciones, periodistas y particulares participan ya en ellas de un modo activo, intensivo y entusiasta.

Desde una perspectiva más concreta, estas normativas permiten a una empresa fijar el tono, las expectativas y las reglas de juego respecto a lo que espera de sus empleados en estos medios. Como cualquier mecanismo de autorregulación, tienen ventajas e inconvenientes (Herrera-Damas, 2011). Entre las ventajas, minimizan el factor sorpresa y materializan la responsabilidad que debe asumir cualquier institución. Entre sus inconvenientes, pueden estar redactadas por editores que desconozcan la naturaleza social y personal de las redes sociales, pueden ir demasiado lejos, emplearse sólo como arma arrojadiza para justificar decisiones exageradas, y capitalizar todos los esfuerzos de la empresa a la hora de acoger esas redes. Resulta evidente que la más impecable de estas normativas no pasaría de ser un simple papel mojado si no fuese acompañada por una estrategia integral, sostenible, permanente y comprometida con las redes sociales y sus posibilidades.

A pesar de todo, consideramos conveniente que los medios se doten de este tipo de políticas. Básicamente por dos razones:

- por el crecimiento que las redes sociales van a seguir experimentando en los próximos años;

- porque, bien empleadas, las redes son herramientas formidables para producir información, distribuirla y reforzar el contacto con la comunidad.

Dicho de otro modo: su llegada es una de las mejores cosas que le ha pasado el periodismo moderno en los últimos años. Darles la espalda sería un error. De todos modos es importante precisar que no creemos en cualquier normativa sino que ésta debería tener unos rasgos específicos (Herrera-Damas, 2011).

\section{Ideas recurrentes en las normativas de medios de prestigio}

Con el propósito de inspirar el diseño de nuevas normativas -varias de las cuales se encuentran en este momento "en construcción"-, este texto pretende detectar los principales consensos en los que coinciden las primeras social media policies de varios medios, entre los que se encuentran algunos de los más prestigiosos. Para ello se ha realizado un análisis cualitativo del contenido ${ }^{5}$ de 22 normativas, con el fin de identificar las indicaciones más recurrentes. Las políticas analizadas son las de AP (Associated press), American Society of News Editors (ASNE), BBC, Bloomberg, Charlotte observer, Freedom communicationS, Los Angeles times, NPR (National public radio), Orlando sentinel, Politico, Reuters, Rockford register star, Saint Louis post-dispatch, Source Media Group, The Denver post, The guardian, The journal gazette, The Manhattan Mercury, The New York times, The Roanoke times, The Wall Street journal y The Washington post. Aunque advertimos diferencias en cuanto al nombre de la normativa, a sus destinatarios, a las redes a las que se refieren, e incluso respecto a la consideración misma de las redes sociales, un examen más detallado nos permite advertir varias coincidencias que identificamos con nuestras propias palabras. Se presentan de forma esquemática en la figura 1 y en la tabla 1.

Las normativas de actuación en redes sociales son documentos que recogen directrices con las que una institución espera regular el comportamiento de sus empleados en ellas

\subsection{El sentido común no es suficiente}

Con su mera existencia, todas las normativas certifican que la progresiva extensión de las redes sociales exige una respuesta, y que apelar al sentido común no es suficiente. Por eso, el afán de regular el comportamiento de los periodistas se encuentra en la esencia misma de las normativas, pese a que éstas prefieran subrayar que nacen para "guiar" y "ayudar" a los profesionales. Encontramos este propósito incluso en aquellas más "a favor" de las redes sociales, como la de NPR. Las de The Wall Street journal y ASNE aluden también de modo expreso a esta insuficiencia del sentido común.

\subsection{Antes o después, tus mensajes serán públicos}

Las normativas analizadas comparten la convicción de que muchos de estos espacios favorecen un tipo de comunicación que, eventualmente, será pública y permanente, certeza que conviene asumir cuanto antes. La de $A P$, por ejemplo, recuerda que esto es así incluso aunque los tuits ${ }^{6}$ hayan sido borrados, ya que pueden haber sido retuiteados por otros usuarios. La de NPR añade que esta publicidad es independiente incluso de la voluntad de su autor. Cada vez resulta más difícil separar las esferas personal y profesional, con el consiguiente riesgo de que la primera pueda comprometer a la segunda. Encontramos referencias a este asunto en 15 de las 22 normativas analizadas, que insisten a sus periodistas en que sean cautos, ya que todo lo que se diga en clave personal puede ser (mal) interpretado en clave profesional.

\subsection{Las redes sociales tienen ventajas e inconvenien- tes}

Algunas de las normativas analizadas, las menos, parten de una visión positiva y entusiasta de las redes sociales (NPR, Bloomberg, Rockford register star y Los Angeles times). Otras, por el contrario, tienen una percepción mucho más 


\begin{tabular}{|c|c|c|c|c|c|c|c|c|c|c|c|}
\hline & $\begin{array}{c}\text { The New } \\
\text { York } \\
\text { times }\end{array}$ & $\begin{array}{c}\text { Los } \\
\text { Angeles } \\
\text { times }\end{array}$ & $\begin{array}{c}\text { The } \\
\text { Roanoke } \\
\text { times }\end{array}$ & \begin{tabular}{|c|}
$\begin{array}{c}\text { Rockford } \\
\text { register } \\
\text { star }\end{array}$ \\
\end{tabular} & Bloomberg & NPR & $\begin{array}{c}\text { The } \\
\text { Denver } \\
\text { post }\end{array}$ & $\begin{array}{l}\text { Orlando } \\
\text { sentinel }\end{array}$ & $\begin{array}{c}\text { St Louis } \\
\text { post } \\
\text { dispatch }\end{array}$ & $\begin{array}{c}\text { Wall } \\
\text { Street } \\
\text { journal }\end{array}$ & $\begin{array}{l}\text { Source } \\
\text { Media } \\
\text { Group } \\
\end{array}$ \\
\hline $\begin{array}{l}\text { El sentido común } \\
\text { no es suficiente }\end{array}$ & & & & & & $x$ & & & & $x$ & \\
\hline $\begin{array}{l}\text { Antes o después } \\
\text { tus mensajes serán } \\
\text { públicos }\end{array}$ & & $x$ & $x$ & & $x$ & $x$ & $x$ & $x$ & $x$ & $x$ & $x$ \\
\hline $\begin{array}{l}\text { Visión más bien } \\
\text { positiva de las } \\
\text { redes sociales }\end{array}$ & & $x$ & & $x$ & $x$ & $x$ & & & & & \\
\hline $\begin{array}{l}\text { Visión más bien } \\
\text { negativa de las } \\
\text { redes sociales }\end{array}$ & & & & & & & & & & & $x$ \\
\hline $\begin{array}{l}\text { Visión ambivalente } \\
\text { de las redes } \\
\text { sociales }\end{array}$ & $x$ & & $x$ & & & & $x$ & $x$ & $x$ & $x$ & \\
\hline Ten espíritu crítico & & & $x$ & & $x$ & & & $x$ & & & \\
\hline Verifica tus fuentes & & $x$ & $x$ & $x$ & $x$ & $x$ & & $x$ & & & $x$ \\
\hline $\begin{array}{l}\text { Evita expresar tu } \\
\text { opinión }\end{array}$ & $x$ & $x$ & $x$ & & $x$ & $x$ & $x$ & $x$ & $x$ & $x$ & $x$ \\
\hline $\begin{array}{l}\text { No publiques } \\
\text { decisiones internas } \\
\text { del medio }\end{array}$ & & & $x$ & $x$ & $x$ & $x$ & $x$ & & & $x$ & \\
\hline $\begin{array}{l}\text { No publiques } \\
\text { detalles del } \\
\text { proceso de } \\
\text { producción de la } \\
\text { información }\end{array}$ & & & $x$ & $x$ & $x$ & & & & & $x$ & \\
\hline $\begin{array}{l}\text { En caso de duda, } \\
\text { consulta }\end{array}$ & $x$ & & $x$ & $x$ & $x$ & $x$ & $x$ & $x$ & $x$ & $x$ & $x$ \\
\hline $\begin{array}{l}\text { En caso de error, } \\
\text { rectifica }\end{array}$ & & & & & $x$ & & $x$ & & & & \\
\hline $\begin{array}{l}\text { Identifícate como } \\
\text { periodista }\end{array}$ & & $x$ & $x$ & $x$ & $x$ & $x$ & $x$ & $x$ & $x$ & $x$ & $x$ \\
\hline $\begin{array}{l}\text { Atribuye el } \\
\text { contenido }\end{array}$ & & & $x$ & $x$ & $x$ & $x$ & & $x$ & & & \\
\hline $\begin{array}{l}\text { Sé íntegro y } \\
\text { ejemplar... y que } \\
\text { lo parezca }\end{array}$ & $x$ & $x$ & $x$ & $x$ & $x$ & $x$ & $x$ & $x$ & $x$ & $x$ & $x$ \\
\hline $\begin{array}{l}\text { Sé sensible y } \\
\text { respetuoso }\end{array}$ & $x$ & & $x$ & $x$ & & & & & & & $x$ \\
\hline $\begin{array}{l}\text { Ojo con las } \\
\text { exclusivas }\end{array}$ & & & $x$ & & $x$ & $x$ & $x$ & $x$ & & & $x$ \\
\hline $\begin{array}{l}\text { Interactúa, pero de } \\
\text { manera profesional }\end{array}$ & & & $x$ & $x$ & $x$ & & $x$ & & & $x$ & \\
\hline
\end{tabular}

Tabla 1

negativa (Freedom communications, Source Media Group y Reuters). Reuters, por ejemplo, ni siquiera enumera sus posibles beneficios. Entre ambos grupos encontramos normativas (15 de las 22 analizadas) con una visión ambivalente respecto a las redes sociales, a las que reconocen ventajas pero también inconvenientes.

Entre los aspectos más apreciados figuran su ubicuidad, su potencial para recoger información, para interactuar con la audiencia, expandir y promocionar el impacto del propio trabajo, y ser más transparence respecto al proceso de producción de la información. Se valoran también las posibilidades que ofrecen para encontrar ideas, localizar fuentes y como oportunidades imaginativas para nuevas formas de periodismo sugerentes.

Entre las críticas se reprocha que consuman tiempo, que puedan distraer a los periodistas de sus labores obligadas, su potencial menoscabo a la propiedad de la información o que faciliten una difusión que escape de la audiencia a la que se pretendía llegar inicialmente. 6 normativas alertan sobre lo fácil que resulta responder o republicar material del propio medio o de otros medios antes de haber reflexionado sobre las consecuencias, y también sobre el posible empleo del material publicado para desacreditar al periodista. Por encima de todas estas críticas, se detecta el lógico temor de los editores a que la reputación del medio quede comprometida.

\subsection{Muchas de las reglas del quehacer tradicional si- guen vigentes}

Las políticas analizadas recuerdan que muchas de las reglas del quehacer periodístico tradicional continúan vigentes; sobre todo las que tienen que ver con la necesidad de tener espíritu crítico, verificar las fuentes o abstenerse de expresar la propia opinión. Se desaconseja publicitar decisiones internas del medio y detalles relativos al proceso de produc- 


\begin{tabular}{|c|c|c|c|c|c|c|c|c|c|c|c|c|}
\hline & Guardian & Reuters & $A P$ & $B B C$ & $\begin{array}{c}\text { Washington } \\
\text { post }\end{array}$ & $\begin{array}{l}\text { Charlotte } \\
\text { observer }\end{array}$ & $\begin{array}{c}\begin{array}{c}\text { The } \\
\text { journal } \\
\text { gazette }\end{array} \\
\end{array}$ & \begin{tabular}{|c|} 
The \\
$\begin{array}{c}\text { Manhattan } \\
\text { Mercury }\end{array}$ \\
\end{tabular} & $\begin{array}{c}\text { Freedom } \\
\text { communications }\end{array}$ & Político & ASNE & Total \\
\hline $\begin{array}{l}\text { El sentido común } \\
\text { no es suficiente }\end{array}$ & & & & & & & & & & & $x$ & 3 \\
\hline $\begin{array}{l}\text { Antes o después } \\
\text { tus mensajes serán } \\
\text { públicos }\end{array}$ & & & $x$ & & $x$ & $x$ & & & $x$ & $x$ & $x$ & 15 \\
\hline $\begin{array}{l}\text { Visión más bien } \\
\text { positiva de las } \\
\text { redes sociales }\end{array}$ & & & & & & & & & & & & 4 \\
\hline $\begin{array}{l}\text { Visión más bien } \\
\text { negativa de las } \\
\text { redes sociales }\end{array}$ & & $\mathrm{x}$ & & & & & & & $x$ & & & 3 \\
\hline $\begin{array}{l}\text { Visión ambivalente } \\
\text { de las redes } \\
\text { sociales }\end{array}$ & $x$ & & $x$ & $\mathrm{x}$ & $\mathrm{x}$ & $x$ & $\mathrm{x}$ & $\mathrm{x}$ & & $\mathrm{X}$ & $x$ & 15 \\
\hline Ten espíritu crítico & & $x$ & & & & & & & & & & 4 \\
\hline Verifica tus fuentes & & & $x$ & & & & & & & & $x$ & 9 \\
\hline $\begin{array}{l}\text { Evita expresar tu } \\
\text { opinión }\end{array}$ & $x$ & $x$ & $x$ & $x$ & $\mathrm{X}$ & $x$ & $x$ & & $x$ & $\mathrm{x}$ & $x$ & 20 \\
\hline $\begin{array}{l}\text { No publiques } \\
\text { decisiones internas } \\
\text { del medio }\end{array}$ & & & & & $x$ & & $x$ & & $x$ & & $x$ & 10 \\
\hline $\begin{array}{l}\text { No publiques } \\
\text { detalles del } \\
\text { proceso de } \\
\text { producción de la } \\
\text { información }\end{array}$ & & $\mathrm{x}$ & & & $\mathrm{X}$ & & $x$ & & $\mathrm{x}$ & & & 8 \\
\hline $\begin{array}{l}\text { En caso de duda, } \\
\text { consulta }\end{array}$ & & $x$ & $\mathrm{x}$ & $\mathrm{x}$ & $\mathrm{x}$ & & & & $x$ & & $x$ & 16 \\
\hline $\begin{array}{l}\text { En caso de error, } \\
\text { rectifica }\end{array}$ & & & $x$ & & & & & & & & $x$ & 4 \\
\hline $\begin{array}{l}\text { Identifícate como } \\
\text { periodista }\end{array}$ & & $x$ & $x$ & $\mathrm{x}$ & $\mathrm{X}$ & & & $x$ & & & $x$ & 16 \\
\hline $\begin{array}{l}\text { Atribuye el } \\
\text { contenido }\end{array}$ & $x$ & $x$ & $\mathrm{x}$ & & & & & & $x$ & & $x$ & 10 \\
\hline $\begin{array}{l}\text { Sé íntegro y } \\
\text { ejemplar... y que } \\
\text { lo parezca }\end{array}$ & $x$ & $x$ & $X$ & $\mathrm{X}$ & $\mathrm{x}$ & & & $\mathrm{x}$ & $x$ & & $\mathrm{x}$ & 19 \\
\hline $\begin{array}{l}\text { Sé sensible y } \\
\text { respetuoso }\end{array}$ & $x$ & & $x$ & $x$ & $x$ & & & & $x$ & & $x$ & 10 \\
\hline $\begin{array}{l}\text { Ojo con las } \\
\text { exclusivas }\end{array}$ & & $x$ & $x$ & $X$ & & & & & & $X$ & $X$ & 11 \\
\hline $\begin{array}{l}\text { Interactúa, pero de } \\
\text { manera profesional }\end{array}$ & $X$ & $x$ & $x$ & $X$ & & & & $X$ & & & $X$ & 11 \\
\hline
\end{tabular}

ción. Los procedimientos para actuar en caso de duda, de error, o ante una exclusiva guardan también muchas similitudes con los del periodismo "clásico".

a) "Ten espíritu crítico"

Para Reuters es esencial que el periodista no pierda sus facultades críticas tampoco en estos espacios. Las políticas de Bloomberg, Orlando sentinel y The New York times insisten en equilibrar los planteamientos en sus coberturas.

b) "Verifica tus fuentes"

En The elements of journalism, Kovach y Rosenstiel recuerdan que la verificación es una de las características identitarias inequívocas de la profesión. 9 normativas aluden a la importancia de esta práctica como un procedimiento eficaz para no incrementar el ruido, sobre todo en un entorno que permite a tantas voces hablar al mismo tiempo. Para ello, los periodistas deben ser escépticos e incrédulos con la veracidad de la información que circula en la Web. c) "Evita expresar tu opinión"

20 de las 22 normativas analizadas recomiendan que los periodistas sean precavidos, eviten dar la impresión de que apoyan una causa concreta y se abstengan de expresar su opinión, una práctica muy frecuente entre los periodistas en Twitter (Lasorsa; Lewis; Holton, 2012) ${ }^{7}$. En este punto encontramos cierta traslación del concepto de imparcialidad que ha sido un rasgo inequívoco de la identidad periodística (Kovach; Rosenstiel, 2007) y que comenzó ya a verse desafiado con la llegada de los blogs (Carlson, 2007; Robinson, 2007; Singer, 2007; Lowrey; Mackay, 2008). La normativa de Los Angeles times es concisa pero muy firme a este respecto: “De la misma manera que fuera de lo digital se prohíben las pegatinas en los coches o los rótulos en el césped, también en la Red se deben evitar las expresiones partidistas". Por lo demás, esta directriz ha sido muy contestada por profesionales y académicos (Buttry, 2009; Siegler, 2009; Ingram, 2009; McLellan, 2009; Parr, 2009; Arrington, 


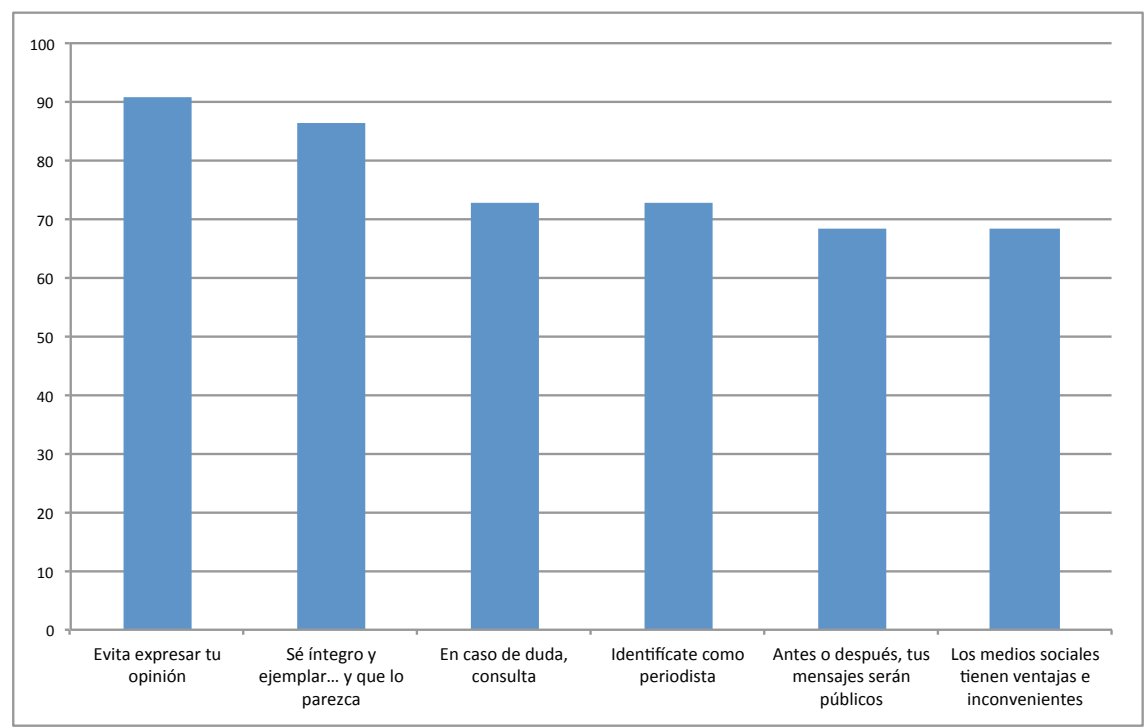

Figura 1. Indicaciones recurrentes en las normativas de actuación en redes sociales de medios de prestigio (\%)

2010, por ejemplo).

Como mucho, las normativas permiten publicar una exención de responsabilidad en la que se diga que su opinión no tiene por qué coincidir con la del medio (Source Media Group). Incluimos aquí también la prohibición de prescribir productos o servicios, algo en lo que insisten las políticas de The Wall Street journal, Orlando sentinel y The New York times.

d) "No publiques decisiones internas del medio"

10 normativas son contrarias a que los periodistas publiquen este tipo de información ya que "podría comprometer la integridad del proceso editorial" (ASNE).

e) "No publiques detalles del proceso de producción"

8 policies se oponen a que se difundan detalles sobre el proceso de producción de la información. En la de The Wall Street journal se indica: "Deja que la cobertura hable por sí misma y no ofrezcas detalles sobre cómo conseguiste, escribiste o editaste una información".

f) "En caso de duda, consulta"

En este caso, 14 normativas recomiendan preguntar al supervisor, a un editor experimentado (Rockford register star) o al editor de social media (Denver post). La de The Wall Street journal vuelve a ser muy escueta pero firme: "Lo más importante a la hora de tratar estas cuestiones es: en caso de duda, consulta...".

g) "En caso de error, rectifica"

4 normativas aluden a la posibilidad de que los periodistas cometan errores, tal como ocurre en los medios tradicionales. Las recomendaciones son: asumir, borrar y publicar la corrección con diligencia, análogamente a lo que encontramos en los códigos deontológicos tradicionales para estos casos.

h) "Ojo con las exclusivas"

11 de las 22 políticas desaconsejan adelantar exclusivas a través de Twitter, ante el temor de quedarse sin ellas o de perder el tráfico hacia el propio sitio. Para evitarlo, mejor ofrecer primero la exclusiva a través de las webs y, sólo después, enlazar vía redes sociales.

i) "Interactúa, pero de manera constructiva"

10 normativas recomiendan a sus periodistas interactuar con la audiencia de un modo profesional, evitando "intercambios prolongados de tira y afloja con gente enfadada, que se van volviendo cada vez menos constructivos". La de la BBC previene también de que se dé la impresión de una interactividad que luego no es tal.

\section{5. "La ética tradicional tam- bién sigue en vigor"}

Junto a modos de proceder clásicos, las normativas recuerdan la validez de la ética tradicional, cuyos principios serían aplicables a estos nuevos espacios. La de Los Angeles times lo tiene claro: "Los métodos y los medios pueden cambiar, pero nuestras normas no". Encontramos recordatorios similares en ASNE, Los Angeles times, $N P R$, Orlando sentinel, St. Louis post-dispatch, Source Media Group, The Roanoke times, The Wall Street journal y The Washington post. Desde una perspectiva más concreta, se alude sobre todo a las siguientes cuestiones:

a) "Identifícate como periodista"

16 policies prescriben que sus periodistas se identifiquen como tales, evitando identidades falsas para conseguir información. La del Rockford register star añade: "Explica a tus contactos en qué estás trabajando, por qué y cómo vas a utilizar la información que te ofrecen. Aclara que toda la información puede ser utilizada y atribuida". Las de Bloomberg y Reuters mencionan el concepto más general de transparencia.

\section{Desde 2009 estas normativas son cada vez más frecuentes en los medios tradi- cionales, ante el temor de que la espon- taneidad de los periodistas comprometa la reputación del medio}

\section{b) "Atribuye correctamente el contenido"}

Las normativas de ASNE, Bloomberg, Freedom communications, NPR, Orlando sentinel, Reuters, Rockford register star, The guardian y The Roanoke times obligan a que sus periodistas atribuyan el contenido que obtienen de la Red de un modo conciso, claro y transparente. Este concepto de transparencia es una de las normas más aceptadas en el periodismo digital (Singer, 2007; Karlsson, 2010; Philips, 2010; Van-der-Wurff; Schönbach, 2011). Puesto que dar crédito a la fuente original forma parte del ethos de las redes sociales. Las de $A P$ y $B B C$ aconsejan extremar las precauciones al republicar contenido en Twitter, para que quede siempre 
claro si el periodista habla a título personal o no.

c) "Sé íntegro y ejemplar... y que lo parezca"

19 de las 22 normativas analizadas insisten en esta necesidad. ¿Los motivos? La certeza de que la integridad es un valor esencial (Orlando sentinel) que no ha cambiado en este nuevo escenario ( $A S N E$, Los Angeles times), el carácter público de lo que se publica online (Los Angeles times, St. Louis post-dispatch) y la posibilidad de que cualquier producto quede asociado a la reputación del medio (The Manhattan Mercury, The New York times). Esto obliga a tener especial precaución con las "apariencias" a la hora de "hacerse amigo" o "seguir" la actividad de determinados grupos (ASNE, Bloomberg, Freedom communications, Los Angeles times, Orlando sentinel, St. Louis post-dispatch, Source Media Group, The Denver post, The Roanoke times, The Wall Street journal) por los prejuicios que esto pueda generar entre la audiencia y también porque las acciones mejor intencionadas pueden ser malinterpretadas (The Roanoke times). La normativa de $N P R$, una de las más entusiastas respecto al uso de las redes sociales, advierte también de este peligro. En ocasiones se recomienda a los periodistas "jugar al despiste" y seguir a instituciones antagónicas para minimizar este riesgo.

\section{Ten cuidado: antes o después tus men-} sajes serán públicos

\section{d) "Sé considerado y respetuoso"}

Igual que los códigos deontológicos, varias normativas $(A P$, ASNE, BBC, Freedom communications, Rockford register star, Source Media Group, The guardian, The New York times, The Roanoke times y The Washington post) obligan a sus profesionales a ser respetuosos también con la competencia (AP y Rockford register star). The guardian prescribe reconocer las aportaciones de los otros, y la $B B C$ decreta respeto con los usos y costumbres del servicio que se emplee "para no dar la impresión de que la $B B C$ se está tratando de imponer a los demás". Esta política recuerda también que a veces será necesario que el medio intervenga para dar pequeños toques de atención a personas que con sus acciones puedan contravenir este principio.

Incluimos también aquí 4 normativas ( $A S N E$, BBC, Rockford register star, The Roanoke times) que llaman la atención para que se tenga especial cuidado en utilizar información publicada por menores o por colectivos que no sean conscientes de que lo publicado en la Red se queda en la Red ${ }^{8}$.

\section{Conclusión}

Los resultados corroboran la existencia de indicaciones recurrentes en las normativas con las que algunos medios de comunicación de prestigio regulan la actuación de sus profesionales en las redes sociales. Sus editores se muestran preocupados ante la posibilidad de que los periodistas empleen estas redes para:

- expresar su opinión;

- mostrar prejuicios y favoritismos;

- ocultar su identidad.
Se recomienda que consulten a un superior, ante la certeza de que las redes hacen posible un tipo de comunicación que antes o después será pública. En el fondo predomina una visión ambivalente de las redes sociales a las que, con sus ventajas e inconvenientes, se reconoce como agentes imprescindibles del nuevo ecosistema mediático.

¿Las redes sociales? Ni buenas ni malas. Depende

\section{Notas}

1. Por recomendación de uno de los revisores, se sugiere "normativas" como la traducción más apropiada para el término inglés "policies". La definición de la RAE para "normativa" -"Conjunto de normas aplicables a una determinada materia o actividad"- resulta, en efecto, pertinente para este contexto.

2. "Guiar" es el eufemismo que a veces utilizan los diseñadores de las políticas. No se trata por tanto de guías con consejos, recomendaciones o pautas de interés para que los periodistas participen de las redes sociales, sino que su objetivo es regular su comportamiento, fijando los límites entre lo lícito y lo que no lo es.

3. Partimos de la definición de redes sociales que aportan Kaplan y Haenlein (2010) "Las redes sociales son un grupo de aplicaciones basadas en internet construidas sobre las bases ideológicas y tecnológicas de la web 2.0 y que permiten la creación e intercambio de contenido generado por usuarios". De una manera más sencilla, estas políticas se refieren sobre todo al uso que los periodistas hacen de las redes sociales y, de un modo especial, al empleo que hacen de Facebook y Twitter.

4. Sobre las utilidades que Twitter, por ejemplo, puede aportar al ejercicio del periodismo se puede consultar Farhi (2009) o, desde un planteamiento más profesional, Rusbridger (2009); o Ahmad (2010) y Hermida (2010), desde una perspectiva más académica.

5. Se puede acceder al contenido de casi todas ellas en el documento ASNE 10 best practices for social media. Hepful guidelines for news organizations, que editan James Hohmann y el Ethics and Values Committee de ASNE, 20102011. Las normativas de $A P$ y de la $B B C$ se pueden encontrar en la dirección:

http://asne.org/Files/pdf/10_Best_Practices_for_Social_ Media.pdf

6. Por recomendación de uno de los revisores, seguimos aquí la sugerencia de la Fundéu que aconseja el término castellanizado "tuiteos".

http://www.fundeu.es/recomendaciones-T-tuitero-tuiteartuiteo-y-retuiteo-terminos-en-espanol-712.html

7. En la explicación de estos autores "Quizá los periodistas tuiteros (j-tweeters) opinan porque microbloguear anima a hacerlo". Aunque no es éste el lugar para explicar esto con más detalle, sí queremos apuntar que es muy frecuente que los periodistas utilicen Twitter para expresar su opinión, tal 
como mencionan los citados autores tras analizar más de 22.000 tuiteos publicados por los periodistas estadounidenses y británicos con más seguidores en esa red. Un $42,7 \%$ del total de periodistas de la muestra expresaron su opinión [sumando las 2 modalidades major opining $(15,7 \%)$ y minor opining (27\%)] (Lasorsa; Lewis; Holton, 2012, p. 29).

8. En este punto, resulta sugerente la propuesta de Whitehouse que defiende la necesidad de actualizar y hacer más explícitos los códigos deontológicos de los medios tradicionales para preservar la privacidad del contenido que se publica online, ante la facilidad para hacerlo y ante la falta de conciencia de ciertos colectivos a este respecto.

Los procedimientos y la ética tradicional todavía siguen vigentes

\section{Bibliografía}

Ahmad, Ali-Nobil (2010). "Is Twitter a useful tool for journalists?" Journal of media practice, v. 11, n. 2, pp. 145-155. http://dx.doi.org/10.1386/jmpr.11.2.145_1

Arrington, Michael (2010). We need more opinion in news, not less.

http://techcrunch.com/2010/07/08/we-need-more-opinionsin-news-not-less

Associated Press (2012). Social media guidelines for AP employees.

http://www.ap.org/Images/Social-Media-Guidelines-7-24-2012 tcm28-8378.pdf

ASNE (2011). 10 best practices for social media. Helpful guidelines for news organizations (editadas por James Hohmann y el Comité de Ética y Valores de ASNE 2010-2011). http://asne.org/Files/pdf/10_Best_Practices_for_Social_ Media.pdf

BBC (2010). Social networking, microblogs and other third party websites.

http://www.bbc.co.uk/guidelines/editorialguidelines/page/ guidance-blogs-bbc-summary

Buttry, Steve (2009). “Objectivity and neutrality aren't the only ways to protect journalist's credibility". The Buttry diary, 4 October.

http://stevebuttry.wordpress.com/2009/10/04/objectivityand-neutrality-arent-the-only-ways-to-protect-journalistscredibility

Carlson, Matt (2007). "Blogs and journalistic authority: the role of blogs in US election day 2004 coverage". Journalism studies, v. 8, n. 2, pp. 264-279.

http://dx.doi.org/10.1080/14616700601148861

Farhi, Paul (2009). "The Twitter explosion". American journalism review, v. 31, n. 3, pp. 26-31.

http://www.ajr.org/article.asp?id=4756

Hermida, Alfred (2010). "Twittering the news: the emergence of ambient journalism". Journalism practice, v. 4, n. 3, pp. 297-308.

http://papers.ssrn.com/sol3/papers.cfm?abstract_ id $=1732598$

Herrera-Damas, Susana (2011). "Social media policies en los medios tradicionales, cuando el sentido común no es suficiente". En Larrondo, Ainara; Meso, Koldo. (coords.) La transformación del espacio mediático, Bilbao, Universidad del País Vasco, pp. 418-436. ISBN: 9788498605716

Ingram, Mathew (2009). "WSJ's Twitter rules: too restrictive". Nieman journalism lab.

http://www.niemanlab.org/2009/05/wsjs-twitter-rulestoo-restrictive

Kaplan, Andreas M.; Haenlein, Michael (2010). "Users of the world, unite! The challenges and opportunities of social media". Business horizons, v. 53, n. 1, pp. 59-68. http://dx.doi.org/10.1016/j.bushor.2009.09.003

Karlsson, Michael (2010). "Rituals of transparency. Evaluating online news outlets' uses of transparency rituals in the United States, United Kingdom and Sweden". Journalism studies, v. 11, n. 4, pp. 535-545.

http://dx.doi.org/10.1080/14616701003638400

Kovach, Bill; Rosenstiel, Tom (2007). The elements of journalism: what newspeople should know and the public should expect. New York: Random House. ISBN: 9780609806913

Lasorsa, Dominic L.; Lewis, Seth C.; Holton, Avery E. (2012). "Normalizing Twitter. Journalism practice in an emerging communication space". Journalism studies, v. 13, n. 1, pp. 19-36.

http://www.academia.edu/548799/Normalizing_Twitter_ Journalism_Practice_in_an_Emerging_Communication_ Space

http://dx.doi.org/10.1080/1461670X.2011.571825

Lowrey, Wilson; Mackay, Jenn-Burleson (2008). “Journalism and blogging: a test of a model of occupational competition". Journalism practice, v. 2, n. 1, pp. 64-81. http://jclass.umd.edu/classes/jour698m/lowrey.pdf http://dx.doi.org/10.1080/17512780701768527

McLellan, Michele (2009). "Washington Post guidelines cast social media as a minefield and that's bad". Knight digital media center. News leadership 3.0, September 29. http://archive.knightdigitalmediacenter.org/leadership_ blog/comments/washington_post_guidelines_cast_social_ media_as_a_minefield_and_thats_bad

Parr, Ben (2009). When does a social media policy go too far? Ask the Associated Press.

http://mashable.com/2009/06/23/ap-social-media-policy

Philips, Angela (2010). "Transparency and the new ethics of journalism". Journalism practice, v. 4, n. 3, pp. 373-382. http://dx.doi.org/10.1080/17512781003642972

Robinson, Sue (2007). "'Someone's gotta be in control here': the institucionalization of online news and the creation of a shared journalistic authority". Journalism practice, v. 1, n. 3, pp. 305-321.

Rusbridger, Alan (2010). "Why Twitter matters for media organisations". The guardian, November 19.

http://www.guardian.co.uk/media/2010/nov/19/alanrusbridger-twitter 
Siegler, MG (2009). Twitter unearths a secret: journalists have opinions.

http://techcrunch.com/2009/09/28/twitter-unearths-ajournalistic-secret-they-have-opinions

Singer, Jane B. (2007). “Contested autonomy: professional and popular claims on journalistic norms". Journalism studies, v. 8, n. 1, pp. 79-95.

Van-der-Wurff, Richard; Schönbach, Klaus (2011). “Bet- ween profession and audience. Codes of conduct and transparency as quality instruments for off- and online journalism". Journalism studies, v. 12, n. 4, pp. 407-422.

http://dx.doi.org/10.1080/1461670X.2010.506055

Whitehouse, Ginny (2010). “Newsgathering and privacy: expanding ethics codes to reflect change in the digital media age". Journal of mass media ethics, v. 25, n. 4, pp. 310-327. http://dx.doi.org/10.1080/08900523.2010.512827

\section{Colección de libros de bolsillo}

\section{EPI-UOC}
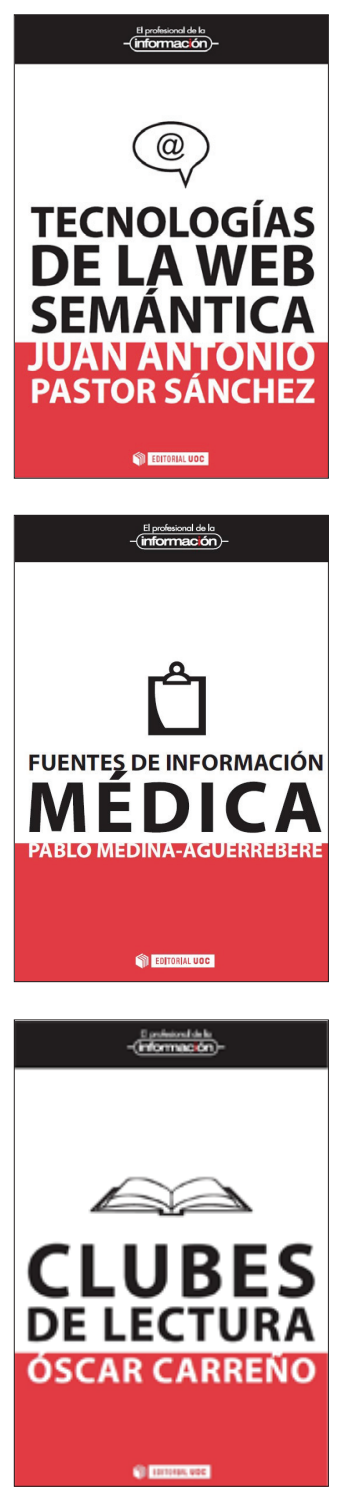
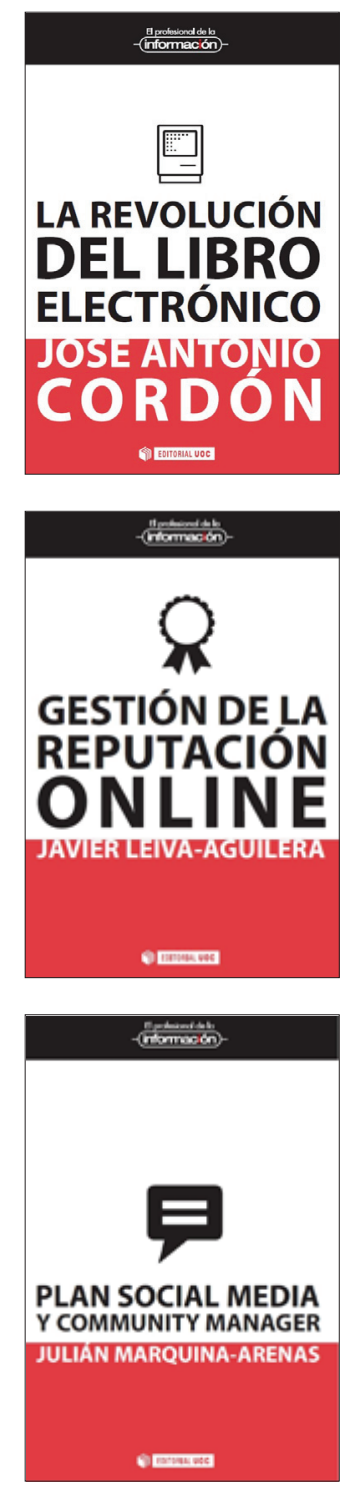
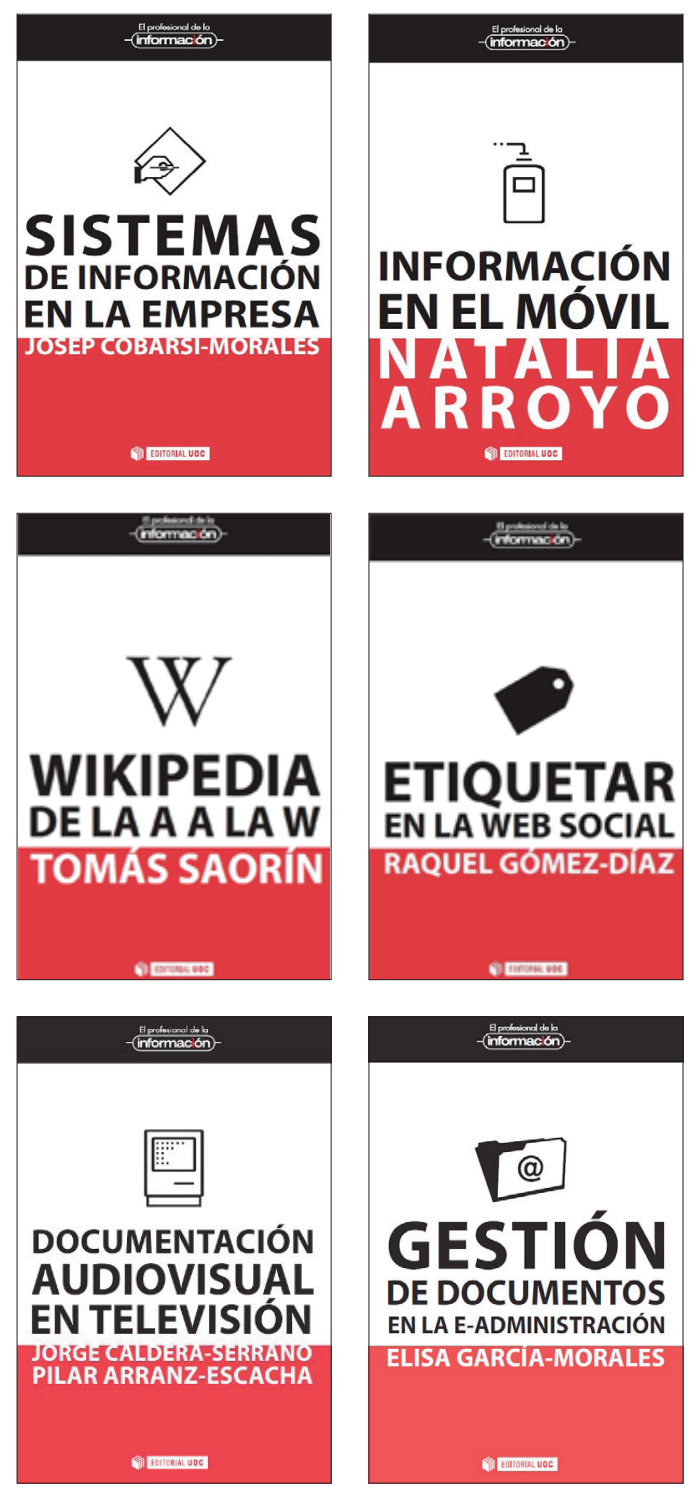

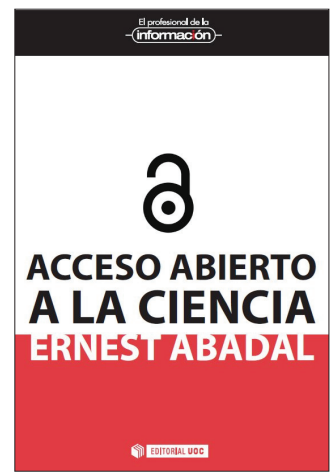

.

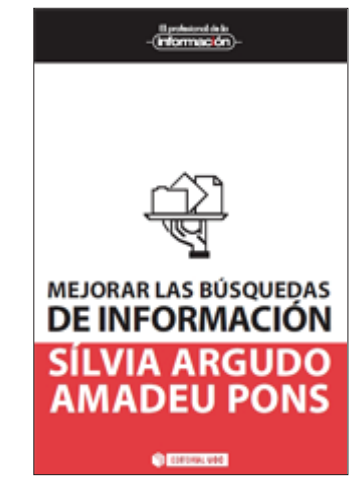

Más información:

http://www.elprofesionaldelainformacion.com/libros.html 